$\Rightarrow$ DISEASE GENOMICS

\title{
Autism sibling differences
}

A new study highlights the genetic heterogeneity of autism spectrum disorder (ASD), even between affected siblings.

More than 100 genetic loci have been associated with susceptibility to ASD. There is a high risk of recurrence of ASD in families, and it was often assumed that siblings with the disorder inherited the same autism-associated mutations.

To understand the genetic factors in familial forms of ASD, Yuen et al. sequenced the whole genomes of 340 individuals (including two affected siblings from 85 families with ASD, as well as both parents). The authors used a medical annotation strategy to classify mutations that were likely to be 'ASD-relevant', including genes previously known to be involved in ASD, candidate and putative risk genes and genes associated with other neurodevelopmental disorders. Analysis of the genomes revealed that $42.4 \%$ (36) of the families carried
ASD-relevant mutations, although in only $31 \%$ (14 of 36 ) of these families did the same rare ASD-relevant mutation occur in both siblings. Of note, the symptoms of the siblings who shared the same ASD-relevant mutations were more similar than those of siblings with different genetic associations.

These findings further emphasize the genetic heterogeneity of ASD, and they have relevance for diagnosis, research and potentially tailoring more individualized treatments. The genome sequences from this study have been made freely available on the Autism Speaks MSSNG database. The aim of the project is to sequence the genomes of 10,000 individuals with ASD.

Bryony Jones

ORIGINAL RESEARCH PAPER Yuen, R. K. C. et al. Whole-genome sequencing of quartet families with autism spectrum disorder. Nature Med. http:// dx.doi.org/10.1038/nm.3792 (2015)

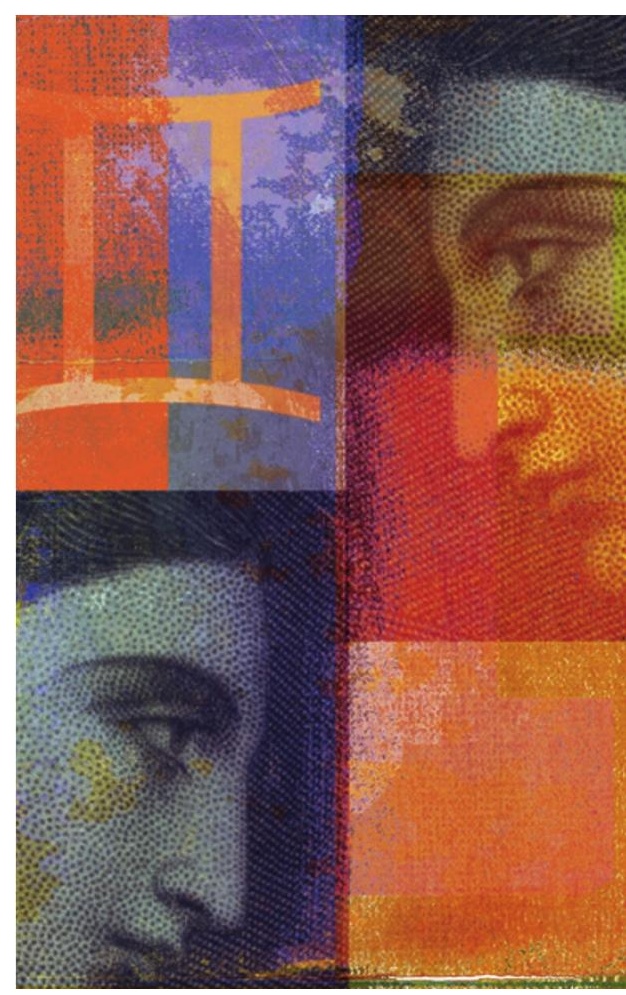

Stephanie Dalton Cowan/Photodisc/Getty 